\title{
Reflets
}

Revue ontaroise d'intervention sociale et communautaire

\section{Les Franco-Ontariens : la résistance comme mode de vie}

\section{David Welch}

Volume 1, numéro 1, printemps 1995

Des pratiques à notre image : défis et ressources

URI : https://id.erudit.org/iderudit/026053ar

DOI : https://doi.org/10.7202/026053ar

Aller au sommaire du numéro

Éditeur(s)

Reflets : Revue ontaroise d'intervention sociale et communautaire

ISSN

1203-4576 (imprimé)

1712-8498 (numérique)

Découvrir la revue

Citer cet article

Welch, D. (1995). Les Franco-Ontariens : la résistance comme mode de vie. Reflets, 1(1), 20-42. https://doi.org/10.7202/026053ar

\section{Résumé de l'article}

Dans un premier temps, ce texte décrit certaines des grandes transformations sociales, économiques et politiques ayant marqué la communauté franco-ontarienne depuis le dix-neuvième siècle, ainsi que certaines des conséquences de ces transformations pour l'intervention sociale et communautaire. Par la suite, l'auteur suggère des pistes de réflexions vers lesquelles la recherche et l'intervention pourraient s'orienter pour mieux s'adapter aux réalités actuelles de cette population.

La toile de fond qui sous-tend cet article se base sur une vision dynamique de la communauté franco-ontarienne, vision qui présente cette population non comme victime, mais plutôt comme actrice engagée dans la transformation de la communauté.
Tous droits réservés (C Reflets : Revue ontaroise d'intervention sociale et communautaire, 1995
Ce document est protégé par la loi sur le droit d'auteur. L'utilisation des services d’Érudit (y compris la reproduction) est assujettie à sa politique d'utilisation que vous pouvez consulter en ligne.

https://apropos.erudit.org/fr/usagers/politique-dutilisation/ 


\section{Les Franco-Ontariens:}

\section{la résistance comme mode de vie}

Dans un premier temps, ce texte décrit certaines des grandes transformations sociales, économiques et politiques ayant marqué la communauté franco-ontarienne depuis le dix-neuvième siècle, ainsi que certaines des conséquences de ces transformations pour l'intervention sociale et communautaire. Par la suite, l'auteur suggère des pistes de réflexions vers lesquelles la recherche et l'intervention pourraient s'orienter pour mieux s'adapter aux réalités actuelles de cette population.

La toile de fond qui sous-tend cet article se base sur une vision dynamique de la communauté franco-ontarienne, vision qui présente cette population non comme victime, mais plutôt comme actrice engagée dans la transformation de la communauté.

\section{David Welch}

Professeur, École de service social, Université d'Ottawa

Les transformations des dernières années dans les services sociaux de langue française en Ontario suscitent un besoin d'identification et d'évaluation des acquis des Franco-Ontariennes et FrancoOntariens dans le contexte des réalités actuelles. Eu égard à la loi 8, la mise sur pied de nouveaux services de langue française et la multiplication d'études sur le sujet, cet article propose une mise au point afin d'orienter nos réflexions, nos débats et nos engagements futurs.

Dans un premier temps, il nous semble essentiel de brosser un tableau de l'histoire franco-ontarienne depuis le $19^{\mathrm{e}}$ siècle en examinant l'impact des grandes transformations ayant marqué la communauté et certaines des conséquences pour l'intervention, en particulier dans le secteur des services sociaux et de la santé. 
Compte tenu de l'orientation de nos recherches antérieures, mais surtout de la pénurie de recherches socio-historiques sur le développement des services sociaux franco-ontariens, plusieurs des exemples utilisés seront tirés du domaine de l'éducation. La création de nos propres institutions de recherche et de publication en service social, apportera des transformations qui s'imposent à ce niveau. Par la suite, nous suggérerons des pistes de réflexion aptes à orienter la recherche et l'intervention de façon à mieux répondre aux défis de la réalité franco-ontarienne actuelle.

La toile de fond sous-tendant ce texte est tissée à partir de trois observations. Premièrement, la communauté est présentement confrontée à des changements sociaux, économiques et politiques de taille qui amènent une remise en question de ses modes de définition comme collectivité. Deuxièmement, depuis les débuts de leur histoire, les Franco-Ontariennes et FrancoOntariens n'ont pas été des victimes passives mais des acteurs engagés dans la transformation de leurs communautés. Enfin, c'est en reconstituant cette histoire collective qu'ils seront en mesure de faire face aux transformations actuelles en leur donnant une nouvelle finalité.

\section{Les Franco-Ontariens: une communauté en constante évolution}

Dans l'étude de l'histoire des Franco-Ontariennes et FrancoOntariens, certains éléments-clé méritent d'être soulignés. D'une part, certaines pratiques (car il y en a d'autres), telles la langue et la religion catholique seront présentées comme des miroirs de la réalité des communautés et étroitement reliées aux changements sociaux, économiques et politiques (Barth, 1969; Juteau-Lee, 1980; Breton, 1984; Hobsbawn et Ranger, 1983; Corrigan et Sayer, 1985). Exprimées d'une multitude de façons par la communauté, les pratiques peuvent s'avérer essentielles à un moment donné pour ensuite devenir d'importance secondaire et 
Reflets

éventuellement disparaitre. Nous en identifierons quelques-unes. Notons que des changements au niveau des pratiques n'entraînent pas nécessairement un changement dans les façons dont une communauté se définit par rapport à elle-même ni par rapport à la société dominante. D'autre part, les frontières entre la communauté minoritaire et le groupe dominant changent à mesure que les rapports sociaux se transforment. Comme nous tenterons de le démontrer, l'isolement n'est pas un facteur essentiel au maintien des différences. Par contre, lorsque les changements dans les rapports sociaux menacent la communauté, les frontières tendent à devenir plus rigides (Siegel, 1970-71) et mieux définies. C'est dans ce contexte que nous présenterons l'attitude et les réactions de la communauté face aux valeurs du groupe dominant comme découlant à la fois de sa résistance aux identités imposées de l'extérieur et des contradictions au sein même de la communauté.

La colonisation canadienne-française en Ontario aux $19^{\mathrm{e}}$ et $20^{\mathrm{e}}$ siècles, en particulier dans le secteur agro-forestier, a d'abord été une reproduction et une extension, aux points de vue économique et social, de la société canadienne-française du Québec. Installés dans des villages relativement homogènes, les Canadiens français déménagés en Ontario ont pu conserver beaucoup de leurs traditions et représentations culturelles et idéologiques. Le développement de l'agro-forestier permettait aux familles de subsister et de demeurer dans une région aussi longtemps que l'agriculture et l'exploitation forestière ont pu co-exister et être rentables (Welch, 1994). D'où une certaine domination des entreprises forestières sur les colons canadiens-français, qui en dépendaient tant pour leurs salaires que pour écouler les produits excédentaires de la ferme, les rendant ainsi fort vulnérables aux aléas de cette industrie (Séguin, 1977:55).

À l'instar de nombreux autres immigrants, les Canadiens français du Québec s'établissent généralement là où leur communauté culturelle est déjà représentée. Par ailleurs, toujours dans un esprit de continuité, les jeunes familles d'agriculteurs qui quittent les régions plus anciennes de l'est de l'Ontario s'établissent souvent dans des régions exploitées plus récemment, tel 
«Bien qu'ils demeurent une minorité dans leur province, les Franco-Ontariennes et Franco-Ontariens ne se perçoivent pas nécessairement comme tel dans leurs milieux, mais plutôt comme faisant partie d'une société canadiennefrançaise plus large.» le Nord-Est, initialement dans les régions de Sudbury et de North Bay. Ces Franco-Ontariennes et Franco-Ontariens ont donc réussi à recréer des frontières culturelles familières, en facilitant l'établissement de leurs proches dans la même région et en reproduisant, dans une large mesure, le mode de vie qu'ils avaient connu ailleurs.

Dans ces différentes régions, la masse critique est généralement suffisante pour fonder des paroisses, des écoles, des organismes sociaux et, plus tard, des coopératives et caisses populaires qui reflètent leur réalité économique et sociale, créant ainsi un réseau plus ou moins autonome de services. Ces institutions constituent des instruments importants dans la conduite de la vie sociale, tout comme dans le contrôle et la distribution des ressources à l'intérieur de la communauté franco-ontarienne. Bien qu'ils demeurent une minorité dans leur province, les FrancoOntariennes et Franco-Ontariens ne se perçoivent pas nécessairement comme tel dans leurs milieux, mais plutôt comme faisant partie d'une société canadienne-française plus large. Cela permet de comprendre le militantisme dont ils ont fait preuve lorsqu'on a voulu imposer des restrictions à leurs institutions, dans le domaine de l'éducation par exemple.

Pour assurer sa protection et son développement, cette nouvelle population de Franco-Ontariennes et Franco-Ontariens a tendance à créer des institutions autonomes ou parallèles qui cherchent à maintenir les particularités culturelles de sa communauté d'origine. C'est ainsi que d'une façon générale ils fondent des écoles de langue française, catholiques ou publiques (Welch, 1993). Certains services tels les hopitaux, les orphelinats, les foyers pour mères célibataires et les hospices pour personnes âgées ont été fondés par des congrégations religieuses et fonctionnent partiellement ou entièrement en français. Avec le temps, plusieurs de ces services, tout en demeurant catholiques, ont été divisés sur une base linguistique.

L'Église catholique franco-ontarienne a été, à beaucoup d'égards, un miroir de l'Église catholique du Québec. Les colons amenent avec eux les pratiques et les rituels qui leur sont traditionnels et familiers. Comme presque toutes ces pratiques 
Reflets

«...l'Église catholique canadienne-française devient à beaucoup d'égards la principale institution de gouvernance de la communauté francoontarienne, de mieux en mieux positionnée pour la transformer à sa propre image.» religieuses et ces rituels sont basés sur la langue française et sur des traditions religieuses, les frontières linguistiques, culturelles et religieuses de la communauté ont été constamment interreliées, renforçant avec le temps la position de l'Église catholique au sein de la communauté.

Graduellement, dans la période suivant la Confédération canadienne, les Franco-Ontariennes et Franco-Ontariens se détachent du Québec, à cause des lois, politiques, pratiques et conjonctures particulières à l'Ontario. Par contre, des Canadiens français du Québec continuent de s'installer en Ontario, notamment dans les villes d'Ottawa, de Cornwall (les usines de "coton»), de Toronto (les manufactures) et dans le Nord-Est ontarien (agroforestier et mines).

Au milieu du $19^{\mathrm{e}}$ siècle, le gouvernement ontarien croyait, naïvement peut-être, que les Canadiennes françaises et Canadiens français accepteraient volontiers de s'assimiler à une culture anglo-britannique jugée supérieure par la majorité anglophone. Quand il devint clair que les Franco-Ontariennes et FrancoOntariens ne s'assimilaient pas mais, qu'au contraire, leur nombre et le pourcentage de la population de langue française augmentaient, ce gouvernement, à partir des années 1880, commence à imposer des restrictions importantes aux écoles françaises, encore majoritairement publiques. Ceci amène les Canadiens français à abondonner les écoles publiques et à fonder de nouvelles écoles séparées catholiques, renforçant le lien entre la langue et la religion et consolidant ainsi l'influence de l'Église catholique au sein de la communauté. Rappelons que dans certains villages de l'Est de la province, les Franco-ontariennes et Franco-Ontariens ont d'abord refusé de transférer leurs taxes vers les écoles séparées, de peur de perdre les avantages fiscaux des écoles publiques (Bourgie et Proulx, 1980; Choquette, 1987; Gaffield, 1987). Avec le temps, l'Église catholique canadienne-française devient à beaucoup d'égards la principale institution de gouvernance de la communauté franco-ontarienne, de mieux en mieux positionnée pour la transformer à sa propre image. Bien qu' elle cherche à promouvoir la vie agraire par le biais de ses premières paroisses et d'autres institutions, l'Église a pu s'adapter à de nouvelles conditions 
économiques amenées notamment par l'urbanisation et l'industrialisation. En même temps, cette position de pouvoir permet à l'Église de continuer d'imposer des points de vue autoritaires et conservateurs sur les diverses institutions francoontariennes et sur la population en général.

\section{Un point tournant: le réglèment XVII}

«Par ses actes de répression, le gouvernement avait contribué à renforcer la communauté franco-

ontarienne et l'influence de l'Église catholique de langue française au sein des diverses institutions socio-politiques.»
Sous-estimant la tenacité et la capacité de résistance passive et active des Franco-Ontariennes et Franco-Ontariens, le gouvernement provincial impose, en 1912, le Règlement XVII qui défend l'usage du français comme langue d'instruction après la deuxième année. Il s'agit d'une tentative claire pour en finir avec l'éducation de langue française en Ontario! Grâce à leur sens d'identité et leur solidarité, les Franco-Ontariennes et FrancoOntariens se mobilisent en s'appuyant sur les institutions existantes. Qui plus est, par le biais de cette résistance, ils créent des institutions autonomes, en éducation par exemple, qui reflètent encore plus que les anciennes écoles bilingues, leurs valeurs et leurs particularités.

Lorsqu'il abolit ce fameux règlement en 1927, 15 ans plus tard, en raison notamment de l'opposition farouche de toute la population canadienne-française au Canada, le gouvernement ontarien se trouve face à une communauté encore plus unie. Par ses actes de répression, le gouvernement avait contribué à renforcer la communauté franco-ontarienne et l'influence de l'Église catholique de langue française au sein des diverses institutions socio-politiques. Le gouvernement est donc obligé de reconnaitre l'existence d'une communauté franco-ontarienne et de limiter ses visées d'assimilation et d'acculturation.

Depuis lors, le gouvernement ontarien garde une présence plus discrète, cherchant par des moyens plus subtils à intégrer la population francophone dans le système scolaire de la province. C'est ainsi que, dans les années suivant l'abolition du Règlement 
Reflets

"Quand les Canadiens français s'installent en Ontario, ils apprennent vite que l'accès à certaines activités économiques leur est impossible.»
XVII, il nomme des inspecteurs franco-ontariens pour superviser les écoles élémentaires, publiques et séparées de langue française. Ces nouveaux agents du ministère de l'Éducation, tout en étant Franco-Ontariens, «parlent» maintenant au nom de l'État. Ils ont la responsabilité de définir les normes éducationnelles d'après les critères établis par le gouvernement, tels le choix des livres et la formation des enseignantes et enseignants. En devenant mieux intégrées au système ontarien, les écoles francoontariennes perdent certaines caractéristiques qui les rendaient culturellement et politiquement différentes.

Nous pouvons constater que pendant ce siècle de colonisation en Ontario, les Canadiennes françaises et Canadiens français ont joué un rôle actif dans le développement économique et politique de la province (Jaenen, 1993, Welch 1994). Cependant, ils accèdent rarement aux postes de direction. Ils sont généralement bûcherons, cheminots semi-qualifiés, petits cultivateurs de subsistance ou travailleuses et travailleurs d'usine. Les formes de capitalisme et l'hostilité de la majorité anglophone les ont exclus des postes de commande dans les secteurs économique et politique, de même que dans beaucoup d'autres institutions. Quand les Canadiens français s'installent en Ontario, ils apprennent vite que l'accès à certaines activités économiques leur est impossible (Thériault, 1982).

Cependant, leurs stratégies économiques et sociales, leur esprit de débrouillardise et les solidarités locales, assurent leur survie en dépit des changements économiques dans une société en effervescence (Berger, 1979:196). Leurs institutions et leur vie socio-économique leur ont permis «de continuer à vivre là où d'autres avaient disparu ou péri» (Berger, 1979:199).

\section{Une période de grands bouleversements}

La période pendant et après la deuxième guerre mondiale transforme complètement le visage économique et socio-culturel de 
«La prolétarisation entraîne progressivement de nouveaux sentiments d'identité... qui viennent s'ajouter à l'identité ethnique existante.»
l'Ontario. Avec une expansion industrielle rapide, l'Ontario accélère sa transformation vers le capitalisme industriel, devenant de plus en plus intégré dans l'économie nord-américaine. Ces changements économiques ont un impact important sur la communauté franco-ontarienne. En effet, en l'espace de quelques années cette population connait un déplacement massif au sein même de la province, de la campagne vers les villes régionales, et du Nord vers les villes industrielles du Sud. Par ailleurs et parallèlement à ces changements démographiques, des milliers de Québécois et d'Acadiens viennent s'installer dans les centres manufacturiers du Sud et dans certaines villes minières du Nord. Finalement, dans les vingt dernières années, un nombre important de personnes parlant le français, venant des quatre coins de la planète, s'est établi principalement dans les centres industriels ou commerciaux de la province.

Avant ces changements rapides, la communauté francoontarienne peut être décrite comme ayant des activités socioéconomiques mi-capitalistes, mi-survivance, formes qui reflètent à la fois sa ruralité et l'influence prépondérante du clergé et des communautés religieuses. La communauté connait rapidement une dépopulation rurale, perdant ainsi une partie importante de sa vie culturelle, à mesure qu'elle s'adapte à la vie et aux valeurs urbaines. Ces changements économiques et démographiques ont un impact important sur les rapports sociaux à l'intérieur de la communauté et sur le sens d'identité de ses membres. La prolétarisation entraîne progressivement de nouveaux sentiments d'identité, par exemple syndicale, qui viennent s'ajouter à l'identité ethnique existante. Éloignés des communautés rurales plus homogènes, les Franco-Ontariennes et Franco-Ontariens forment maintenant une communauté aux allégeances diverses, opposées même, déterminées par les conditions d'une societé civile et urbaine. Pour faire face à ces bouleversements, les gens se sont employés à créer dans les villes, des institutions socioéconomiques intermédiaires (caisses populaires, centres sportifs et communautaires) qui rappellent d'une certaine façon celles de la campagne. La classe ouvrière développe de nouvelles loyautés, basées par exemple, sur des conflits de classe, car elle se retrouve 
Reflets

«...être Canadiennes

françaises et Canadiens français signifie qu'ils continuent de mener une vie pauvre et misérable...» en contradiction avec l'élite qui, à ses yeux, défend des intérêts de moins en moins pertinents. Dans certaines circonstances avant et après la deuxième guerre mondiale, notamment lors de grèves à Sudbury et à Kirkland Lake, la petite bourgeoisie francoontarienne s'allie à la bourgeoisie ontarienne contre les travailleurs franco-ontariens. (Arnopolous, 1982; Pelletier, 1987).

Il est important de noter qu'après la deuxième guerre mondiale, bon nombre de Franco-Ontariennes et Franco-Ontariens, les hommes bien plus que les femmes, voient leur niveau de vie et leurs conditions de travail s'améliorer rapidement, grâce surtout à la montée de la syndicalisation. En général, les salaires grimpent plus rapidement que le coût de la vie, souvent après de longues luttes syndicales (Arnopolous, 1982). Cependant, ce ne sont pas tous les Franco-Ontariennes et Franco-Ontariens qui connaissent une amélioration de leur situation économique à la suite de cette transition. Bien que plusieurs bénéficient de meilleurs salaires, un segment de la population n'a pas les compétences requises pour profiter de la prospérité d'après-guerre; ces gens vivent dans la pauvreté, aussi bien dans les villages que dans les villes, et forment une sorte de "classe défavorisée» sur le plan économique. Plusieurs Franco-Ontariennes et FrancoOntariens, de même que des Québécoises et Québécois et des Acadiennes et Acadiens qui, eux aussi, avaient quitté les régions rurales de leur province pour s'établir dans les villes de l'Ontario, continuent de subir les conséquences de plus de cent ans de dépendance économique. Souvent dépourvus des compétences requises et handicapés par un taux d'analphabétisme élevé, ils trouvent fort difficile, voire impossible, de profiter des perspectives économiques que leur offre la ville et ce, quelle que soit la région. Pour ces gens, être Canadiennes françaises et Canadiens français signifie qu'ils continuent de mener une vie pauvre et misérable qui ne fait que perpétuer les nombreuses contradictions historiques basées sur la langue et la ruralité. En un sens, la «campagne» les avait suivis en ville. 


\section{Impact du politique et de l'économique sur le sens d'identité}

Tous ces changements dans les rapports internes et externes ont un impact sur la perception qu'ont les Franco-Ontariennes et Franco-ontariens de leurs institutions. Quand il existait un consensus au sein de la communauté par rapport aux frontières ethniques, il a souvent été plus facile de se mettre d'accord sur les formes institutionnelles les plus appropriées. Par contre, à mesure que la perception du sens d'identité communautaire, ou de l'importance des diverses pratiques culturelles, telles que la langue et la religion, se diversifie, le consensus par rapport aux types d'institutions les plus appropriés s'avère beaucoup plus difficile. En plus, dans les centres urbains en particulier, les institutions sont souvent imposées de l'extérieur, rendant difficile leur transformation par la communauté locale. Certains Franco-Ontariennes et Franco-Ontariens continuent à lutter pour des institutions parallèles comme moyen de se donner des services et de conserver leur identité et leurs pratiques culturelles.

De même que nous avons tous plusieurs niveaux de conscience, nous nous définissons aussi en fonction de plusieurs identités. L'identité ethnique n'est qu'une des formes d'identification sociale, (Epstein, 1978), qui comprend aussi des identités régionales ou des identités de classes sociales, de sexe, etc. Ainsi, certains Franco-Ontariennes et Franco-Ontariens vivant de nouvelles réalités économiques et sociales se forgent de nouvelles identités basées sur les "critères de réussite» de la majorité anglophone et de ses institutions beaucoup mieux nanties, et renient leurs racines, afin de mieux s'intégrer, en partie ou totalement, à cette majorité.

Par ailleurs, la communauté franco-ontarienne n'est pas isolée des grands changements qui transforment les villes ontariennes et canadiennes dans la période après la guerre. Dans tous les secteurs de la vie socio-économique, les gouvernements deviennent de plus en plus interventionnistes. Au nom des réformes et du 
Reflets

"...les nouvelles

banlieues toujours en

expansion et

majoritairement

anglophones ont

détruit en partie

certaines concentrations

historiques de Franco-

Ontariennes et

Franco-Ontariens...» bien-être de la société, le gouvernement ontarien tansforme la structure et le contenu des institutions et les quartiers francoontariens. Par exemple, plutôt que de venir en aide aux diverses communautés dans les quartiers populaires en leur fournissant des services locaux, les divers paliers de gouvernements appuient la démolition de vieux quartiers, au nom de la rénovation urbaine et d'une meilleure gestion municipale. Dans ce procédé, ils forcent le dépaysement ou l'expropriation de milliers de personnes, et détruisent une multitude de quartiers ethno-culturels (Stasiulis, 1980:24-25). Cette nouvelle forme d'intervention étatique a un effet très négatif sur certains quartiers franco-ontariens. Ottawa, par exemple, une partie de la Basse-Ville et toutes les plaines LeBreton sont démolies, obligeant les Franco-Ontariens à déménager plus à l'est dans les banlieues d'Ottawa ou bien de l'autre côté de la rivière des Outaouais (Carrière, 1983). Suite à ces rénovations, les nouveaux arrivants dans la Basse-Ville sont souvent des anglophones, de classe moyenne ou à faible revenu, logés dans les HLM. Les transformations autour du Marché By, un ancien quartier d'affaires où se retrouvent aussi écoles et lieux de culte pour les Franco-Ontariennes et Franco-Ontariens, ont eu le même effet. Ce manque de respect de l'intégrité ethno-culturelle s'est également manifesté à Sudbury, avec la destruction d'une bonne partie du quartier "Moulin à Fleur», de même qu'à Toronto dans les quartiers autour de l'ancienne paroisse du Sacré-Coeur. Dans les banlieues des trois villes en question, où se retrouvent les plus grandes concentrations de Franco-Ontariennes et FrancoOntariens, la création de nouveaux espaces d'expression socioculturelle s'avère difficile, à cause de la dispersion de la population, de la plus grande distance des écoles et des centres communautaires. En plus, les nouvelles banlieues toujours en expansion et majoritairement anglophones ont détruit en partie certaines concentrations historiques de Franco-Ontariennes et FrancoOntariens, telles à Orléans, Tecumseh près de Windsor, et dans les villages autour de Sudbury. De nouvelles pratiques culturelles sont souvent centrées autour des intérêts de la classe moyenne, laissant moins de place à la culture ouvrière qui occupait une place d'honneur dans les anciens quartiers à majorité franco-ontarienne. Cette dispersion a peut-être apporté un niveau de vie plus élevé 
sur le plan économique mais cette amélioration a entraîné des changements dans l'identité socio-culturelle.

\section{Le rôle des femmes dans une communauté en mutation}

"Souvent cantonnées an foyer et aux soins qu'elles doivent prodiguer aux enfants et aux personnes âgées, les femmes sont de plus en plus isolées sur le plan social.»
La socialisation des jeunes enfants franco-ontariens a souvent été assurée par les femmes, aidées des grands-parents. Ainsi les femmes, dans leur rôle d'éducatrice et de mère, contribuent non seulement à préserver l'ethnicité mais également à la reproduire en transmettant les pratiques culturelles et en préparant les jeunes à s'adapter aux transformations continuelles dans la socitété (Juteau-Lee et Robarts, 1981:19). Le sens d'identité de la jeunesse francoontarienne s'est également façonné à travers les interactions avec les membres de sa famille élargie, et avec l'entourage plus ou moins proche. Dans ces échanges sociaux, les enfants francoontariens apprennent progressivement à s'identifier à ceux qui font partie de leur collectivité. Les frontières sont maintenues principalement par le biais des relations domestiques ou privées et soutenues par des réseaux externes ou publics (écoles, institutions, centres communautaires).

Par contre, avec la croissance de l'industrialisation et de l'urbanisation, le rôle des Franco-Ontariennes s'amenuise, notamment dans les sphères économique et sociale, ce qui entraîne des répercussions sur leur statut au sein de la famille. Souvent cantonnées au foyer et aux soins qu'elles doivent prodiguer aux enfants et aux personnes âgées, les femmes sont de plus en plus isolées sur le plan social. L'État prend progressivement la relève d'un grand nombre de tâches qu'elles avaient l'habitude d'accomplir et exige des titres ou des diplômes que la plupart d'entre elles ne possèdent pas. Dans certaines régions comme le Nord-Est, les emplois les mieux payés se trouvent dans les mines et dans l'industrie forestière, lieux interdits aux femmes. Ces dernières se retrouvent donc surtout dans des emplois peu rémunérateurs du 
Reflets

secteur des services, tels la restauration, les ménages, les services sociaux et de santé, etc. Ceci augmente leur dépendance financière par rapport aux hommes. L'urbanisation et l'augmentation des salaires ont donc amélioré la situation des hommes canadiens français mais non celle des femmes (Proulx, 1982, Coulombe, 1985). À mesure que les familles émigrent vers la ville et que les enfants et petits-enfants côtoient de plus en plus la culture dominante, la tâche des femmes devient encore plus difficile; dans certains cas, elles se sentent coupables du fait que leurs enfants aient perdu l'usage du français.

\section{Évolution récente des lois et politiques}

Les années 1960 ont amené des progrès dans la reconnaissance des droits des Franco-Ontariennes et Franco-Ontariens, notamment les lois 140 et 141 qui reconnaissent le droit à des écoles élémentaires et secondaires de langue française. La Loi sur les langues officielles du gouvernement fédéral (1969) amorçe, par ailleurs, un processus de bilinguisme dans les institutions du gouvernement fédéral.

Au nom de la modernité, les gouvernements investissent des millions de dollars dans la transformation des services de santé et des services sociaux dans la province. En outre, le gouvernement provincial facilite la construction de nouveaux hôpitaux, remplaçant les institutions locales, souvent fondées et gérées par des religieuses canadiennes-françaises. Le déménagement de l'Hôpital général d'Ottawa de la Basse Ville, un quartier franco-ontarien, vers une banlieue, majoritairement anglophone, constitue un exemple de ces déplacements. Les Franco-Ontariennes et Francoontariens profitent, au même titre que les autres, de meilleurs services et de professionnels mieux qualifiés. Néanmoins, même si elles assurent certains bénéfices matériels aux professionels et à

la communauté, ces améliorations imposées pas l'État viennent affaiblir le sens d'identité franco-ontarienne. Graduellement, les 
"...la communauté

franco-ontarienne se trouve bien souvent confrontée à des services et des professionnels qui ne sont pas représentatifs de leur communauté.» communautés se sentent incapables de s'identifier à d'autres institutions, à d'autres formes de gouvernance, basées sur des valeurs socio-politiques différentes. Dans la foulée de modernité, les Franco-Ontariennes et Franco-Ontariens ont à payer un prix élevé. Combien de ces nouveaux hôpitaux se trouvent dans ou près des quartiers francophones, comme c'était souvent le cas avec les anciens hôpitaux locaux? Dans quelle mesure ces services sociaux ou de santé peuvent-ils fournir un personnel qualifié de langue française, capable de saisir les nuances et les particularités de la communauté franco-ontarienne? (Pettey et Ouimet, 1988). Avec ces interventions du gouvernement provincial, les Franco-Ontariennes et Franco-Ontariens ont perdu le contrôle d'institutions qu'ils avaient créées et dirigées depuis plusieurs générations. Aujourd'hui, la reprise d'un certain contrôle d'institutions qui autrefois leur appartenaient, et les possibilités de transformation ou de création d'autres organismes exigent beaucoup d'énergie.

Les réformes des années 1960 et 1970 ont permis aux FrancoOntariennes et Franco-Ontariens de profiter d'une certaine mobilité socio-économique et de meilleurs services dans les services sociaux et de santé. Par contre, sans le contrôle d'un État de langue française, comme c'est le cas au Québec, et avec le retrait de l'Église catholique de la plupart des services, la communauté franco-ontarienne se trouve bien souvent confrontée à des services et des professionnels qui ne sont pas représentatifs de leur communauté.

Dans le domaine de l'éducation, où les Franco-Ontariennes et Franco-Ontariens ont mieux fait valoir certains besoins, ces ambiguïtés persistent encore (Welch, 1991-92). La gestion de leurs systèmes scolaires n'a été cédée que parcimonieusement aux régions et aux pouvoirs locaux, et ce, souvent avec un financement inadéquat, comme ce fut le cas à Ottawa, avec le conseil scolaire francophone homogène. Le droit à des écoles secondaires catholiques, découlant de demandes de la communauté catholique de langue anglaise, donne de nouvelles écoles aux Franco-Ontariennes et Franco-Ontariens, mais au prix de divisions majeures au sein de la communauté. 
Reflets

"Historiquement, la majorité des services sociaux ont été gérés par des organismes privés à but non lucratif qui recevaient certains octrois $d u$ gouvernment provincial.»
Dans les secteurs des services sociaux et de la santé, la réalité a été, et demeure encore, différente de celle de l'éducation. Historiquement, la majorité des services sociaux ont été gérés par des organismes privés à but non lucratif qui recevaient certains octrois du gouvernment provincial. Mis à part certains services administrés par des communautés religieuses, les autres services sociaux, tels les sociétés de l'aide à l'enfance, sont administrés par des anglophones, même dans des villes comme Ottawa et Sudbury avec une forte minorité franco-ontarienne. Les services étaient - et sont encore - rarement offerts en français. C'est seulement en 1981 que le gouvernement provincial a commencé à octroyer des fonds pour le développement de services en français (Carrière, 1994).

Aujourd'hui encore, le gouvernment ontarien fournit la majorité des services à la communauté par l'entremise de plus de 2200 organismes privés (Carrière, 1994) généralement unilingues anglophones. La loi sur les Services en français (1986) assure que dans les régions désignées, certains services sociaux et de santé soient fournis en français. La loi 8 est sans contredit un pas en avant important; son application permet un plus grand respect des droits linguistiques des Franco-Ontariennes et FrancoOntariens et a comme effet secondaire la création de centaines d'emplois pour des gens de la communauté au sein des secteurs public et para-public. Par contre, les services sociaux administrés par les municipalités et les organismes non-gouvernementaux, sont exclus des provisions de cette loi. (Boucher, 1994). Même dans les villes qui se sont déclarées bilingues, les services sont souvent mal définis et laissés au bon vouloir de fonctionnaires peu conscients des inégalités de ces services et peu sympathiques aux revendications de la population franco-ontarienne. Au nom de la rationalisation et des coupures dans les dépenses, les postes désignés comme bilingues demeurent souvent gelés, en attente de subventions spéciales de la part du gouvernment provincial ou fédéral.

Même si les organismes peuvent demander une désignation en vertu de la loi 8, seulement 1,8 \% l'ont fait (Carrière, 1994), car le gouvernement accorde peu de fonds pour la mise en place 
de ces services. Il n'est pas surprenant qu'avec le peu de services offerts en français les Franco-Ontariennes et Franco-Ontariens doivent chercher longtemps avant de se faire servir dans leur langue. Souvent ces mêmes services ne sont que des «traductions» de programmes anglophones peu adaptés aux spécificités de la culture franco-ontarienne. Finalement, il existe encore une importante pénurie d'intervenantes et d'intervenants franco-ontariens dans les services sociaux car jusqu'à tout récemment la formation dans les services sociaux et de la santé se faisait uniquement en anglais, alors que les Franco-Ontariennes et Franco-Ontariens ont souvent basé leur choix de carrière en partie sur les options éducatives disponibles en français.

\section{Quelques pistes de réflexion}

"Il importe donc de chercher des voies nouvelles qui aideront... à développer de nouvelles pratiques sociales..."
Aujourd'hui les lois provinciales comme celles sur les services en français ou sur l'éducation accordent aux Franco-Ontariennes et Franco-Ontariens certains droits et une place plus grande dans la gestion des services à la communauté. Néanmoins, la reconnaissance de ces droits les oblige souvent à se battre entre eux pour des ressources qui ont tendance à diminuer. En se situant dans un cadre étroit et légaliste, les Franco-Ontariennes et FrancoOntariens se font définir des pouvoirs qui se situent à l'extérieur de leur communauté, et risquent ainsi de voir ralentir la recherche de nouvelles solutions collectives aux problèmes qui les confrontent. Il devient alors de plus en plus urgent de définir nos besoins et de proposer des solutions avant que d'autres le fassent pour nous.

Il importe donc de chercher des voies nouvelles qui aideront les intervenantes et intervenants franco-ontariens à développer de nouvelles pratiques sociales reflétant la situation et les particularités culturelles de leur communauté.

Les questions et les recherches doivent déborder du cadre imposé par les qualités et les lacunes de la loi 8 sur les services en français, afin que les politiques et programmes puissent dépasser 
Reflets

"Quels seront les effets des changements, des modifications aux politiques d'assurance chômage... sur la population du nord-est ontarien... souvent touchée par le chômage saisonnier?» une simple traduction en français des pratiques sociales anglophones existantes. Trop souvent, les intervenantes et intervenants, particulièrement ceux qui occupent une position de leadership au sein de la communauté, limitent leur analyse aux questions linguistiques sans lier ces préoccupations à des questions plus globales, comme la pauvreté et l'équité...

Ce travail de recherche et de conscientisation prend autant plus d'importance aujourd'hui en raison de la remise en question aux niveaux fédéral et provincial des politiques sociales existantes, qui s'ajoutent aux coupures dans les programmes municipaux. Quels seront les effets des changements, des modifications aux politiques d'assurance chômage (le projet Axworthy) sur la population du nord-est ontarien, une population souvent touchée par le chômage saisonnier? Quelles seront les conséquences des politiques de création d'emplois pour les jeunes travailleuses et travailleurs franco-ontariens? Quels seront les effets des coupures des programmes de l'aide sociale pour les personnes francophones qui cherchent à retourner à l'école et éventuellement sur le marché du travail? Quelles seront les répercussions du refus du gouvernement provincial de subventioner davantage les programmes de garderies pour les familles franco-ontariennes, déjà défavorisées à ce niveau? Quelles seront les conséquences pour les projets franco-ontariens du transfert des projets d'alphabétisation populaire au Conseil ontarien de la formation de la main d'oeuvre (COFAM)? Et finalement quelles répercussions les nouvelles politiques en immigration auront-elles sur les néo-Franco-Ontariennes et néo-Franco-Ontariens venus au Canada comme réfugiés?

Ces questions invitent un approfondissement de nos réflexions non seulement sur la nature de la recherche et des pistes d'intervention mais aussi sur les façons de faire de la recherche et de l'intervention. Les études de besoin effectuées en contexte minoritaire au cours des dernières années ont privilégié les méthodes de recherche de type quantitatif.Ainsi, suite à des enquêtes auprès «d'experts» dans la communauté (souvent des professionnels), les chercheurs ont dressé des listes de lacunes dans la prestation de plusieurs services français. Les «besoins de la communauté», sont souvent présentés comme si certaines normes globales existaient au 
"Une recherche plus approfondie de type ethnographique... nous permettrait de mettre en lumière certains aspects jusqu'ici occultés, comme... des systèmes de débrouillardise des populations minoritaires francophones de l'Ontario.» sein d'une communauté franco-ontarienne homogène. Les particularités liées aux classes sociales, aux rôles sexuels, aux spécificités régionales sont occultées dans de telles analyses. Il est maintenant nécessaire d'ajouter une autre dimension à ces études «par des recherches d'orientation ethnographique, les seules à pouvoir réellement donner la parole aux communautés régionales...» (Corin, et al.,1990:31). Basée sur des principes anthropologiques, cette forme de recherche, liée à l'intervention, permettra de découvrir certains aspects de la réalité qui sont moins apparents, et de mettre en relief les valeurs, symboles et mythes qui finissent par donner un sens aux pratiques des Franco-Ontariennes et FrancoOntariens. Par exemple, quand une Franco-Ontarienne violentée par son mari frappe à la porte d'un refuge anglophone, est-ce parce que les services en français n'existent pas, ou parce qu'elle ne tient pas à des services dans sa langue, ou encore pour des raisons plus complexes que la langue et la culture? Sa demande de services en anglais manifeste peut-être un désir de garder l'anonymat, la peur que "le village» soit au courant de sa situation. Une recherche plus approfondie de type ethnographique, liée à l'intervention, nous permettrait de mettre en lumière certains aspects jusqu'ici occultés, comme les contraintes des "pratiques silencieuses» ou des systèmes de débrouillardise des populations minoritaires francophones de l'Ontario. Une telle approche pourrait inspirer des solutions plus réalistes, axées sur les réalités locales, et faciliter la définition de nouvelles formes d'intervention sociale. Cela permettrait de mieux mettre en valeur un des paradoxes de la culture franco-ontarienne, où la dépendance côtoie une grande créativité (Corin et al., 1990:39).

Dans l'exploration de nouvelles pistes de recherche et d'intervention, basées sur le local et sur l'identité culturelle, il faudrait non seulement reconnaitre les grands écarts socio-économiques qui existent au sein de la communauté franco-ontarienne, mais aussi encourager les pratiques qui luttent contre ces disparités. Tout en dénonçant l'exploitation économique que les FrancoOntariennes et Franco-Ontariens ont subie de la part de la majorité anglophone, il faut admettre qu'une certaine partie du leadership franco-ontarien a non seulement profité de cette exploitation, 
Reflets

"...une certaine partie du leadership francoontarien a non seulement profité de cette exploitation, mais s'est également montrée indifférente aux conditions de vie dans les milieux populaires.» mais s'est également montrée indifférente aux conditions de vie dans les milieux populaires. Et que dire du rôle du clergé et des communautés religieuses face à de tels contextes de disparité? Notons, par exemple, que les premières initiatives en alphabétisation populaire dans les années 1980 sont venues de la base et non des organismes porte-parole, tels l'Association canadiennefrançaise de l'Ontario (ACFO). De façon générale, jusqu'à présent, les revendications économiques prônées par «nos» leaders se sont limitées au type «esprit d'entreprise», avec peu d'attention aux projets économiques communautaires. Ces initiatives sont généralement encouragées par des intervenantes et intervenants qui travaillent depuis des années dans les milieux populaires, souvent éloignés des pouvoirs décisionnels au sein de la communauté et en marge des grands circuits socio-économiques. Ces intervenantes et intervenants locaux se sont rendu compte que l'avenir socio-économique était intimement lié non seulement à la création d'emplois, mais aussi à un nouveau partage du pouvoir au sein des communautés.

Il importe aussi de conjuguer les intérêts culturels aux enjeux liés à la classe, au sexe et à la race, car, en Ontario français comme dans la société dominante, ce sont souvent les hommes d'origine européenne et de niveau socio-économique moyen ou supérieur qui gèrent les intérèts des populations et qui parlent en leur nom. Souvent les organismes franco-ontariens officiels semblent concevoir le développement des services sociaux de langue française comme étant une affaire "pour les pauvres» quand en réalité la mise sur pied de nouvelles garderies, de services de soins de longue durée, par exemple, sont une réponse à des besoins réels qui touchent tous les secteurs de leur communauté.

La communauté franco-ontarienne continue aujourd'hui à se transformer. Il est important de ne pas en exclure des groupes, souvent minoritaires, qui contribuent à son développement et à son essor. Plutôt que de se cantonner dans des modèles existants, la revendication du droit à la différence linguistique et culturelle doit s'étendre pour inclure tous les domaines de la vie culturelle, économique, sociale et politique de la communauté. Trop souvent, par désir de protéger les droits âprement acquis, les Franco-Ontariennes 
"...il est essentiel

d'éviter de recréer et de reproduire en français les mêmes aliénations et exclusions qui ont fait partie de notre histoire de peuple minoritaire.» et Franco-Ontariens perçoivent toute forme de dissidence comme une attaque contre les valeurs historiques de leur communauté. En adoptant des politiques axées sur le pluralisme interne, la francophonie ontarienne sera en mesure de transformer les frontières socio-politiques et d'élargir ses rangs aux autres qui partagent les mêmes préoccupations. Certains progrès ont été réalisés au niveau de l'intégration des personnes d'expression française de différentes communautés ethno-culturelles. Par contre, jusqu'à quel point les Franco-Ontariennes et Franco-Ontariens sont-ils prêts à remettre en question les structures et les orientations de leurs organismes afin de permettre une meilleure participation, mais surtout de mieux partager le pouvoir de direction? La communauté franco-ontarienne doit aussi trouver des moyens pour inclure ceux et celles qui ont été exclus de ses institutions, en éducation par exemple. Nous faisons référence ici entre autres aux décrocheurs et aux personnes analphabètes. Dans toute intervention auprès des membres de la communauté, il est essentiel d'éviter de recréer et de reproduire en français les mêmes aliénations et exclusions qui ont fait partie de notre histoire de peuple minoritaire.

Dans les années à venir, il sera important de poursuivre la recherche d'une plus grande autonomie dans nos rapports avec la majorité anglophone et au sein de nos propres organismes et institutions, en faisant valoir les intérêts de toute la communauté, plutôt que ceux des élites, qu'elles soient franco-ontariennes, anglophones, ou autres... La question reste posée: qui sont les alliés de la population franco-ontarienne, en cette fin de vingtième siècle, dans sa quête d'identité? Les Franco-Ontariennes et Franco-Ontariens doivent chercher des alternatives qui enrichiront les pratiques existantes, même quand les intervenantes et intervenants réussissent à travailler principalement ou exclusivement en français, car la culture n'est pas simplement une question de langue.

Dans cette recherche d'autonomie, d'alternatives et d'un plus grand respect des particularités de la communauté francoontarienne, il faut constamment chercher à identifier et articuler une vision commune afin de pouvoir «forger un discours sur ce 
Reflets

qu'il y a de valeurs communes à travers les différences multiples qui tissent le monde moderne» (Blais, 1993). Cette capacité de comprendre nos différences doit être liée à une conscience de la nécessité d'unir nos interventions et nos revendications avec ceux et celles qui recherchent comme nous une société construite sur des nouvelles formes de solidarité.

\section{Conclusion}

Dans ce texte, nous avons présenté le tableau historique d'une communauté dont les identités et les frontières ethno-culturelles se sont transformées au rythme des grands changements sociaux, économiques et politiques de la province et du pays, tout en situant les Franco-Ontariennes et Franco-Ontariens au centre de leur propre histoire, comme un peuple capable de façonner son destin.

Loin de se présenter comme une relique du passé, ce sens d'identité contient une vitalité qui nous aidera à affronter les grands changements que nous vivons présentement dans la societé en général et dans nos différents champs d'intervention en particulier. Malgré des exclusions importantes qui peuvent exister au sein de leur communauté, les Franco-Ontariennes et FrancoOntariens demeurent des personnes au centre de ces changements, et non pas en marge, ou folklorisés, car ils forment une communauté historique, avec de longues traditions et une capacité de transformer leurs rapports avec l'État ou avec les autres forces dominantes. La création comme entité de la communauté franco-ontarienne au $18^{\mathrm{e}}$ siècle et son existence encore aujourd'hui font partie des grandes transformations que le Canada a connues. Leurs revendications au cours de l'histoire ont toujours recherché plus que «de simples revendications culturelles pour la diversité.» (Melucci, 1993).

Par contre en cherchant à saisir l'essentiel de cette histoire, et en explorant des pistes nouvelles, il demeure essentiel de ne pas 
"C'est dans la recherche de nouvelles alliances dans un contexte identitaire élargi que nous pourrons construire de nouvelles solidarités." oublier que les diverses composantes du leadership FrancoOntarien ont privilégié, à beaucoup d'égards, le maintien du statu quo, sauf par rapport à la sauvegarde de la langue française et une certaine culture franco-ontarienne. L'Église catholique, malgré toutes ses bonnes oeuvres, a travaillé activement à transformer la communauté franco-ontarienne en fonction de sa propre image, tout en imposant des points de vue autoritaires et conservateurs sur les diverses institutions franco-ontariennes. L'élite franco-ontarienne, tout en se présentant comme le défenseur de la communauté, s'est souvent mise en contradiction avec des secteurs de la communauté en défendant des intérêts peu pertinents à leurs yeux ou même carrément en opposition aux leurs. Finalement l'État, tout en étant forcé de limiter ses visées d'assimilation et d'acculturation, et en accordant certains droits et une participation dans la gestion de certains services, continue néanmoins à définir des formes acceptables d'identité francoontarienne.

L'analyse du contexte historique et social de la réalité francoontarienne révèle que les diverses résistances se sont organisées dans la communauté (et non autour de l'élite et de l'État) et que c'est ainsi qu'elles continuent de se manifester. C'est dans la recherche de nouvelles alliances dans un contexte identitaire élargi que nous pourrons construire de nouvelles solidarités.

\section{Bibliographie}

ARNOPOULOS, Sheila, Mcleod (1982). Hors du Québec point de salut?, Montréal, Libre Expression. BARTH, Fredrik (1969). Ethnic Groups and Boundaries, London, George Allen \& Unwin. BERGER, John (1979). Pig Earth, London, Writers and Readers Publishing Cooperative.

BLAIS, L. (1993). L'étrangère à nos portes: femmes, pauvreté et santé mentale, Montréal, Université de Montréal, thèse de Ph.D.

BOUCHER, Lee-Ann (1994). Analyse socio-historique des politiques ontariennes de prestation des services sociaux en français (travail présenté à l'École de service social dans le cadre du cours SVS 5510 donné par David Welch et Michèle Kérisit), Ottawa, École de service social, Université d'Ottawa.

BOURGIE, Francine et Jean-Pierre PROULX (1980). Embrun, Ottawa, l'Association des enseignantes et enseignants franco-ontariens de l'Ontario.

BRETON, R. (1984). «The Production and Allocation of Symbolic Resources: An Analysis of the Linguistic and Ethnocultural Fields in Canada», Canadian Review of Sociology and Anthropology, vol. 21, no 2, pp. 124-144. 
CARRIÈRE, Fernand (1983). Le confort... sans histoires: les Franco-Ontariens, de 1960-1970, Toronto, TV Ontario.

CARRIÈRE, Richard (1994). «La loi 8 et les services sociaux destinés aux familles francophones», Colloque Familles francophones - Multiples réalités à Sudbury 3, 4,5 novembre 1994, Sudbury.

CHOQUETTE, Robert (1987). La foi, gardienne de la langue en Ontario 1900-1950, Montréal, Bellarmin.

CORIN, Ellen E., Gilles BIBEAU, Jean-Claude MARTIN et Robert LAPLANTE (1990). Comprendre pour soigner autrement, Montréal, Les Presses de l'Université de Montréal.

CORRIGAN, Philip et Derek Sayer (1985). The Great Arch: English State Formation as Cultural Revolution, Oxford, Basil Blackwell.

COULOMBE, D. (1985). «Doublement ou triplement minoritaire», Revue de l'Université d'Ottawa, vol. 55, no 2, pp. 131-136.

EPSTEIN, A.L. (1978). Ethos and Identity, London, Tavistock Publications.

GAFFIELD, Chad (1987). Language Schooling and Cultural Conflict, Kingston et Montréal, Presses de l'Université McGill et Queen's.

HOBSBAWN, E. et T. RANGER, éd. (1983). The Invention of Tradition, Cambridge, Cambridge University Press.

JAENEN, Cornelius J. (1993). Les Franco-Ontariens, Ottawa, Les presses de l'Université d'Ottawa.

JUTEAU-LEE, Danièle, (1980). «Français d'Amérique, Canadiens, Canadiensfrançais, FrancoOntariens, Ontarois: Qui sommes-nous?», Pluriel, vol. 24, pp. 21-42

JUTEAU-LEE, Danièle et Barbara ROBERTS (1981). «Ethnicity and Femininity: (d') après nos expériences», Revue canadienne des études ethniques, vol. 8, no 1, pp. 1-23.

MELUCCI, Alberto (1993). «Vie quotidienne, besoins individuels et action volontaire», Sociologie et Sociétés, vol. 25, no 1, pp. 189-197.

PELLETIER, Jean-Yves (1987). Le conflit minier, Toronto, TVOntario.

PETTEY, Donna et OUIMET, Renée (1988). Quand je suis malade, je ne suis pas bilingue. Ottawa, Association canadienne pour la santé mentale, Section d'Ottawa-Carleton.

PROULX, P. (1982). La part des femmes il faut la dire, Ottawa, La Fédération des femmes canadiennesfrançaises.

SÉGUIN, Normand (1977). La conquête du sol au $19^{\text {ìme }}$ siècle, Montréal, Les Éditions du Boréal express.

SIEGEL, Bernard J. (1970-1971). «Defensive Structuring and Environment Stress», American Journal of Sociology, vol. 76, pp. 11-32.

STASINLIS, Daiva (1980). «The Political Structuring of Ethnic Community Action: A Reformulation?», La revue canadienne des études ethniques, vol. 12, no 3, pp. 19-43.

THÉRIAULT, J.Yvon (1982). «Trois évidences sur notre réalité économique», Colloque national en économie, Ottawa, FFHQ.

WELCH, David (1991-1992). «La lutte pour les écoles secondairesfranco-ontariennes», Revue du Nouvel-Ontario, no 13-14, pp. 109-131.

WELCH, David (1993). "Early Franco-Ontarian Schooling As a Reflection and Creator of Community Identity» Ontario History, vol. 85, no 4, pp. 321-347.

WELCH, David (1994). «Formes socio-économiques et identité franco-ontarienne dans le nordest de l'Ontario. Étude socio-historique de développement économique», Égalité, no 34, automne, pp. $46-80$. 